\title{
Prehospital Thrombolytic Therapy Prior to Percutaneous Coronary Intervention a Comparative Study with Conventional Approach in Acute ST-segment Elevation Myocardial Infarction
}

\author{
Hussein Shaalan MD \\ Ain Shams University - Cardiology Department
}

\begin{abstract}
Background: early revascularization post MI is the corner stone in the therapy of acute myocardial infarction. Primary PCI proved itself in the management of STEMI with patency rate more than $90 \%$.However, not every hospital has PCI facility. New thrombolytic agent (tenecteplase) is now available which can be given easily outside hospital (Prehospital) to facilitate PCI and preserve the cardiac muscle.
\end{abstract}

Aim of the work: comparing primary PCI results in patients with STEMI when tenecteplase was given prior to intervention as early as possible (Prehospital), with those who had only PCI without thrombolytic therapy.

Patients and Methods: the presenting study included 60 patients, divided into two equal groups, group I patients received tenecteplase followed by primary PCI and group II underwent primary PCI only in acute STEMI. 12 leads ECG, cardiac enzymes, echocardiographic study, and coronary angiography were done for all patients. PCI results were recorded in addition to immediate and 6 month follow up. All patients received the same adjuvant medical therapy (aspirin, clopidogrel, heparin and tirofiban if needed).

Results: both groups showed non significant differences in peak cardiac enzymes, ejection fraction before and after PCI, and angiographic success post PCI. The hard end points (death, MI) did not show statistical difference between both groups both inhospital and on follow up. However group I showed significant difference $(\mathrm{P}<0.05)$ in more direct stenting, less procedure time, and more recurrence of chest pain post PCI compared with group II. There were highly significant differences $(\mathrm{P}<0.001)$ in favor of group I in more TIMI 3 flow, less thrombus burden, and less pathological Q waves; and in favor of group II in more clinical success post PCI without complications. The call to balloon interval was shorter in group II $(\mathrm{P}<0.05)$. No major bleeding was seen in both groups.

Conclusion: facilitated PCI has the advantages of decreasing thrombus burden in acute STEMI, decreasing procedure time and achieving more TIMI 3 flow; however the hard end points are not different from primary PCI and the recurrence of chest pain is significantly more with facilitation. Tenecteplase use is not associated with major bleeding before PCI.

KEY words: tenecteplase facilitated PCI, Prehospital thrombolysis.

\section{Introduction}

Acute ST-segment elevation myocardial infarction (STEMI) is a potentially life threatening cardiovascular emergency. Early revascularization of the infarct related artery is required to salvage the myocardium at risk. Thrombolytic agents have the advantage of being given early after the onset of chest pain either in the ambulance or inside the coronary care units of nearby hospitals (Morrison et al., 2000).
New thrombolytic agents such as tenecteplase (metalyse) are given as bolus IV dose and do not need long infusion time needed for other agents like streptokinase (SK) or rt-PA (ASSENT)-3 Investigators 2001.

However, primary percutaneous coronary intervention (PCI) in acute myocardial infarction has a higher patency rate of the infarct related artery with better 
myocardial salvage if done in the proper time window (Nallamothu et al., 2003).

It is recommended that the call to needle time interval is better not exceeding 90 minutes to achieve good results, and the door to needle not exceeding 20 minutes. The door to balloon time interval should not exceed 60 minutes (De luca et al., 2003).

Whatever the methods of revascularization, many adjuvant therapeutic agents are increasingly used nowadays such as clopidogrel, and glycoprotein IIb/IIIa inhibitors in addition to heparin (unfractionated or low molecular weight heparin), aspirin and IV nitrates. These agents help to facilitate and maintain revascularization (Montalescot et al., 2001).

Initially, primary PCI within 48 hours after intake of thrombolytic agents did not show encouraging results and the deferred approach was preferred (Ellis et al., 2000).

After the discovery of the new thrombolytic agents that show more fibrin specificity, less bleeding, fewer allergies, less homodynamic affection and superior efficacy, primary PCI can play a complementary role in achieving complete and maintained revascularization compared with thrombolysis alone.(Dubois et al., 2003).

\section{Aim of the work}

Evaluation of efficacy and safety of giving new thrombolytic agents (tenecteplase) before primary PCI in comparison with conventional primary PCI alone in acute STEMI

\section{Patients and methods}

Sixty patients were included in this study, 30 patients (group I) received tenecteplase prior to primary PCI in acute STEMI and the other 30 patients (group II) received conventional primary PCI approach. Tenecteplase (metalyse) was given as IV bolus dose according to patient body weight, preceded by heparin UFH or LMWH and aspirin as shown in table 1.

Clopidogrel $300 \mathrm{mg}$ was given in addition to the aspirin and heparin before
PCI as pretreatment before stenting and thereafter by $75 \mathrm{mg}$ daily.

IV tenecteplase can be given at home or inside the ambulance or inside CCU of primary hospital before reaching the referral hospital where primary PCI can be carried out.

\section{Inclusion Criteria:}

(1) All patients with documented STEMI (presented in the eligible time window ( $\leq 12$ hours from onset of chest pain or new onset LBBB). (2) Young patients $(<55 y)$ are strongly recommended. (3) Patients living far away from the hospital with $\geq 90$ min transportation time and (4) unstable patients with hypotension, severe intolerable pain, serious life threatening arrhythmias or arrest are best candidates for TNK+ PCI (Facilitated PCI).

\section{Exclusion Criteria:}

- Patients who have contraindication for fibrinolytic therapy such as recent hemorrhagic diatheses, cerebral hemorrhage, perforated peptic ulcer or advanced diabetic retinopathy, and severe uncontrolled hypertension. These patients will undergo primary PCI without antecedent fibrinolysis.

- All patients with unsuitable anatomy for PCI or in need of emergency CABG.

\section{Methods:}

All patients are subjected to: full history taking and clinical examination laying stress on:

Major risk factors (age, gender, smoking, diabetes, hypertension, (HTN), dyslipidemia and positive family history of CAD).

- Past history of ischemic episodes (angina, myocardial infraction (MI), previous catheterization (PCI) or CABG.

- History of major bleeding diathesis.

- History suggestive of compromised LV function.

- Evaluation of hemodynamic and vital signs (pulse, blood pressure, respiratory rate), signs of cardiac 


\section{Hussein Shaalan MD}

decompensation and pulmonary congestion.

- 12-Leads surface ECG for documentation of STEMI, site of injury, rhythm disorders, and conduction delay.

- Echocardiographic evaluation of cardiac function, valvular insufficiency, intracavitary clots or ruptured septum or pericardial effusion. Laboratory exa-minations for evidence of myocardial injury (CK, CK MB) Liver Function tests (PT, SGOT and SGPT), CBC, (Hb, Platelet count), blood sugar, renal function (To avoid contrast induced nephropathy), electrolyte and lipid profile. All laboratory tests are done in the hospital.

Tenecteplase (Metalyse, Boehringer Ingelheim). This fibrinolytic agent was chosen for Prehospital thrombolysis as it is given as bolus injection not by long infusion time as in rt- PA, and not associated with immune reaction or hypotension. This agent is also fibrin specific and not associated with major bleeding from the puncture site after PCI. It has a high patency rate and more prevalent TIMI III flow post fibrinolysis. The maximum benefit of tenecteplase is expected to be after 20 minutes and its half life is short (2-hours). IV tenecteplase can be given at home, inside the ambulance or in the CCU. Heparin (UFH) is given before tenecteplase by $100 \mathrm{u} / \mathrm{kg}$. Aspirin 325 to $500 \mathrm{mg}$ IV or oral. Clopidogrel $300 \mathrm{mg}$ orally then once daily. The decision of giving fibrinolytic therapy before PCI is subjected to the evaluation of the physician either in the ambulance or in the CCU before referral to the tertiary hospital in which primary PCI will be preformed. Nitroglycerine SL or IV is given for pain relief or control high blood pressure or left ventricular dysfunction.

Other adjuvant therapy like beta blockers and morphine or ACE inhibitors are given for control of heart rate, blood pressure or LV dysfunction. Glycoprotein
IIb/IIIa inhibitors such as tirofiban (aggrastat) are given when there is evidence of failed thrombolysis such as persistent or recurrent chest pain or during PCI and thereafter when there is heavy thrombus burden. It is given according to body weight and following the instructions of use made by the manufacturer. It is given inside the CCU.

\section{Catheter Procedure:}

Diagnostic coronary angiography is done first using standard Judkin's technique.

$5 \mathrm{~F}$ sheaths and catheters are the standard size to start with and $6 \mathrm{~F}$ sheaths and catheters are used only if more support is needed or vessel canulation failed. LV injection is occasionally done before coronary angiography. The left coronary artery is the first to be studied followed by the right coronary artery. Low osmolar non ionic contrasts (imeron) are used in all patients.

The culprit vessel is identified and if PCI will be done the case will be included in the study. In case the patient is not candidate for PCI or emergency $\mathrm{CABG}$ is needed the case is excluded.

\section{PCI Procedure}

Floppy guide wire (PT-grafix Bostonscientific) 0.014 was used to canulate the infarct related artery in all patients. Then either provisional or direct stenting were done according to the suitable anatomy. Only one vessel intervention for the culprit artery is done but occasionally more than one vessel PCI is done according to the operator opinion if the patient is unstable. Adjuvant medications such as intracoronary nitroglycerine, adenosine, dipyridamole, nitroprusside or tirofiban are given to help recanalization and to manage no reflow if the thrombus burden is big according to the individual case and operator decision.

Sometimes deep catheter intubation is used to help balloon or stent deployment or to aspirate large clots. Patients received intracoronary tirofiban are kept on the same medicine for 24 hours IV post PCI. Bare metal stents (BMS) are usually used and sometimes drug eluting stents (DES) are 
preferred especially for young patients and for diabetics. Low profile balloons such as viva or maverick (Boston scientific) are preferred with $\leq 1: 1$ balloon to vessel ratio and for stents 1: 1 stent: vessel ratio is used.

\section{Post PCI management:}

Sheath removal was done after $6-8$ hours after checking PTT.

Aspirin is given indefinitely and clopidogrel is given post PCI for at least one month in case of bare metal stents or for $9-12$ months in case of DES. Tirofiban is given for at least 24 hours post PCI in some patients who received it during or before PCI.

Follow up cardiac enzymes, serial 12leads ECG and echocardio-graphic study are done for all patients.

The patients who received tenecteplase plus PCI (facilitated PCI) are compared to those who received only PCI in terms of success rate and complication rate in hospital and in the next 6 months post intervention.

Data were collected, revised, verified then edited on P.C. Data were analyzed statistically using SPSS statistical package version 13. The following tests were done; mean values with standard deviations, number of values with percentage, $T$. test for independent samples and chi square test for qualitative values. $P$ values were considered significant below 0.05 and highly significant below 0.001 .

\section{Results}

This study included 60 patients with acute STEMI. All patients underwent primary PCI, Group I (30 patients) received in addition IV tenecteplase before PCI and Group II (30 patients) did not receive any thrombolytic agents prior to intervention.

The presenting study was carried out in Klinikum Braunschweig, Deutschland in the time interval from January 2004 to April 2005.

The mean age in group I was $55.1 \pm 13$ ranged from $34 \rightarrow 88$ while in group II the mean age was $55.7 \pm 11$ ranged from $30 \rightarrow 78$ ( $\mathrm{P}$ value $>0.05)$
There were $23(77 \%)$ male patients in group I versus $24(80 \%)$ male patients group in II and $7(23 \%)$ female patients in group I versus $6(20 \%)$ female patients in group II ( $\mathrm{P}$ value $>0.05)$.

Recent onset chest pain of acute STEMI was present in 22(73\%) patients in group I versus $20(67 \%)$ patients in group II ( $\mathrm{P}$ value $>0.05$ ).

All Patients had no past history of major bleeding diathesis.

Prehospital thrombolysis was carried out either at home in $8(27 \%)$ patients, inside the ambulance in $12(40 \%)$ patients or in a small volume hospital or primary medical center in 10 (33\%) patients $(\mathrm{P}$ value $>0.05$ ).

Group I included 21(70\%) patients who were found to be stable during STEMI versus 22(73\%) patients in group II (P Value $>0.05)$.

Nine (30\%) unstable patients of group I developed complications during STEMI, 5 patients were hypotensive and 4 patients were successfully resuscitated following cardiac arrest.

Eight (27\%) unstable patients of group II developed complications during STEMI, 5 patients were hypotensive and 3 patients were successfully resuscitated following cardiac arrest ( $\mathrm{P}$ value $>0.05$ ).

Acute PCI in group I was considered rescue when the patients still had chest pain with persistent or recurrent ST-segment elevation despite thrombolysis, 20(67\%) patients underwent rescue PCI versus $10(33 \%)$ patients who underwent facilitated PCI which means that the PCI was done despite no chest pain or ECG changes. In other words PCI in group I was still needed in most of the patients who received tenecteplase.

The mean time interval from onset of chest pain and receiving thrombolysis (the call to needle interval) was $1.93 \pm 1.3$ hours and the call to balloon interval was $8.1 \pm 6.51$ hours, the mean time interval between thrombolysis and PCI was $6.23 \pm 5.19$ hours.

In group II the call to balloon interval was $4.57 \pm 3.3$ hours $(\mathrm{P}$ value $=0.01)$ which is statistically significant. Giving tenecteplase before PCI might overcome the 


\section{Hussein Shaalan MD}

delay in performing PCI especially for patients referred late for intervention. Also, the interval is measured from the time of receiving patient call which is longer than the door to balloon interval by nearly one hour.

Group I showed STEMI in inferior leads in $17(57 \%)$ patients versus $16(53 \%)$ patients in group II, STEMI in anterior leads in $13(43 \%)$ patients versus $14(47 \%)$ patients in group II ( $\mathrm{P}$ value $>0.05$ ). More than $50 \%$ of the study population belongs to the low risk MI patients.

Tirofiban was given in addition to heparin, aspirin and clopidogrel in $13(43 \%)$ patients in group I and in the same number in group II. This means that the only factor for facilitated PCI is tenecteplase.

All patients presented by ST segment elevation MI; however pathological Q waves persisted in $50 \%$ of patients in group $\mathrm{I}$ and in all patients of group II ( $\mathrm{P}$ value $<0.001)$ which is highly significant. However, The EF is not different in both groups as more than $50 \%$ of patients belong to inferior (low risk) MI.

$5 \mathrm{~F}$ sheath and catheters were used in $18(60 \%)$ patients of group I versus $21(70 \%)$ patients of group II ( $\mathrm{P}$ value $>0.05$ ).

In one patient of group I the $5 \mathrm{~F}$ sheath and catheters were used only for diagnosis and the $6 \mathrm{~F}$ sheath and catheters were used for intervention.

In the rest of the patients $6 \mathrm{~F}$ sheath and catheters were used from the start in $11(37 \%)$ patients of group I versus $9(30 \%)$ patients of group II ( $\mathrm{P}$ value $>0.05$ ). patients

Rt. femoral approach was used in all

Simple lesions were present in 18 (60\%) patients in both group I and group II. This means that PCI procedures were matched together regarding the encountered difficulties.

Residual lesions were present in 29 (97\%) patients of group I versus $30(100 \%)$ patients in group II. ( $\mathrm{P}$ value $>0.05$ )

Group I showed 7(23\%) patients with long or multiple lesions in the infarct related artery, 2(7\%) patients with bifurcation lesions and 4(13\%) patients with tortuous course of infarct related artery versus $4(13 \%)$ patients, $5(17 \%)$ patients and $3(10 \%)$ patients of group II respectively. (P value $>0.05$ )

Group I showed thrombus containing lesions in $2(7 \%)$ patients versus $7(23 \%)$ patients in group II ( $\mathrm{P}$ value $<0.001$ ) which is highly significant. Tenecteplase is responsible for this difference as both groups received the same adjuvant therapy including tirofiban.

Balloon predilatation was used in 21 (70\%) patients in group I versus $26(86 \%)$ patients in group II ( $\mathrm{P}$ value $<0.05)$ which is statistically significant.

No significant difference was found between both groups regarding the hard end points (death, MI). Group I showed more significant occurrence of chest pain. However, on follow up no significant difference was found between both groups

Table (1): The dose schedule of Tenecteplase according to body weight

\begin{tabular}{|c|c|c|}
\hline Body weight $(\mathbf{k g})$ & TNKase (mg) & Reconstituted TNKase (mL) \\
\hline$<60$ & 30 & 6 \\
\hline 60 to $<70$ & 35 & 7 \\
\hline 70 to $<80$ & 40 & 8 \\
\hline 80 to $<90$ & 45 & 9 \\
\hline$\geq 90$ & 50 & 10 \\
\hline
\end{tabular}




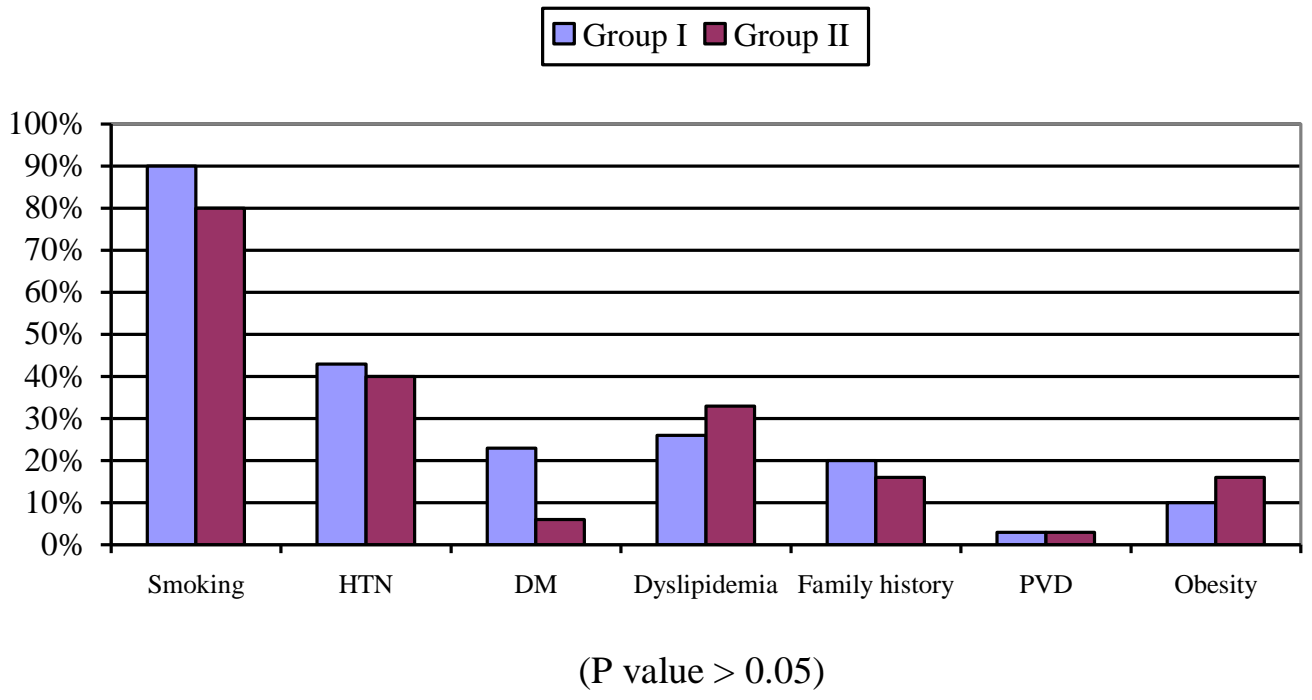

Figure (1): The distribution of major risk factors between both groups. (The two groups were matched)

Table (2): Shows the number of patients showing STEMI in different leads

\begin{tabular}{|l|c|c|c|c|}
\hline \multicolumn{1}{|c|}{ Group } & Antroseptal & Inferior & Extensive anterior & High lateral \\
\hline \multirow{2}{*}{ Group I } & 7 & 17 & 5 & 1 \\
& $(23 \%)$ & $(57 \%)$ & $(17 \%)$ & $(3 \%)$ \\
\hline Group II & $8(27 \%)$ & $16(53 \%)$ & $4(13 \%)$ & $2(7 \%)$ \\
\hline
\end{tabular}

Table (3): Shows mean peak cardiac enzymes (CK-CK MB) among patients of group I and group II

\begin{tabular}{|l|c|c|}
\hline \multicolumn{1}{|c|}{ Group } & CK & CK-MB \\
\hline Group I & $2931 \pm 2826 \mathrm{u} / \mathrm{L}$ & $342 \pm 302 \mathrm{u} / \mathrm{L}$ \\
\hline Group II & $2702 \pm 1364 \mathrm{u} / \mathrm{L}$ & $277 \pm 142 \mathrm{u} / \mathrm{L}$ \\
\hline P-value & $>0.05$ & $>0.05$ \\
\hline
\end{tabular}

Table (4): Shows the mean EF of the patients in both groups before and after PCI

\begin{tabular}{|l|c|c|}
\hline & EF before PCI & EF after PCI \\
\hline Group I & $45.9 \pm 10$ & $51.4 \pm 10$ \\
\hline Group II & $45 \pm 11$ & $49 \pm 11$ \\
\hline P-value & $>0.05$ & $>0.05$ \\
\hline
\end{tabular}

Table (5): Shows the findings of diagnostic coronary angiography.

\begin{tabular}{|l|c|c|c|c|}
\hline \multicolumn{1}{|c|}{ Vessel affected } & LMS & Single vessel & Two vessels & Multi vessels \\
\hline Group I & $2(6 \%)$ & $19(63 \%)$ & $4(13 \%)$ & $7(23 \%)$ \\
\hline Group II & $0(0 \%)$ & $16(53 \%)$ & $8(26 \%)$ & $6(20 \%)$ \\
\hline
\end{tabular}




\section{$\square$ Group I $\square$ Group II}

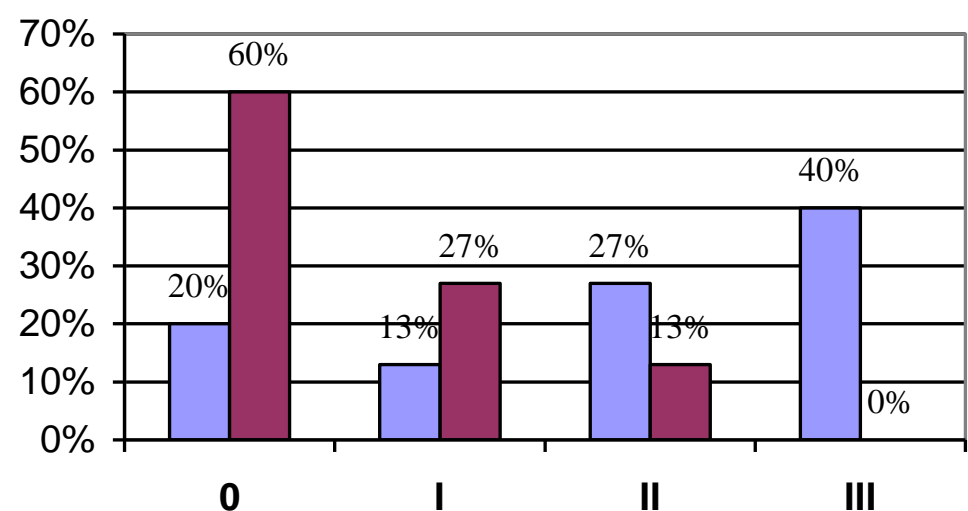

Figure (2): Shows the TIMI flow pattern among the study groups on diagnostic angiography ( $P$ value $<0.001$ ) which is highly significant.

Table (6): shows the distribution of culprit vessels

\begin{tabular}{|c|c|c|c|c|c|c|}
\hline Vessel & LMS & LAD & RCA & LCX & Diagonal & Ramus \\
\hline Group I & $1(3 \%)$ & $9(30 \%)$ & $18(60 \%)$ & $1(3 \%)$ & $1(3 \%)$ & - \\
\hline Group II & $0(0 \%)$ & $15(50 \%)$ & $12(40 \%)$ & $2(7 \%)$ & - & $1(3 \%)$ \\
\hline
\end{tabular}

Table (7): Shows the type of balloon, the size, the length, the inflation time and atmospheric pressure

\begin{tabular}{|c|c|c|c|c|c|c|c|}
\hline \multirow{2}{*}{ Group } & \multicolumn{3}{|c|}{ Type of balloon } & \multirow{2}{*}{$\begin{array}{c}\text { Size } \\
(\mathrm{mm})\end{array}$} & \multirow{2}{*}{$\begin{array}{l}\text { Length } \\
(\mathrm{mm})\end{array}$} & \multirow{2}{*}{$\begin{array}{l}\text { Pressure } \\
\text { (bar) }\end{array}$} & \multirow{2}{*}{$\begin{array}{c}\text { Duration } \\
(\mathrm{sec})\end{array}$} \\
\hline & Maverick & Viva & IQ & & & & \\
\hline Group I & 4 & 17 & - & $\begin{array}{c}2.55 \pm 0.4 \\
\text { range } 2 \rightarrow 3.5\end{array}$ & $\begin{array}{l}20(19) \\
30(2)\end{array}$ & $\begin{array}{c}12.7 \pm 2.1 \\
\text { (range } 8-16 \text { ) }\end{array}$ & $\begin{array}{c}35.6 \pm 10 \\
\text { range }(25 \rightarrow 61)\end{array}$ \\
\hline Group II & 4 & 21 & 1 & $\begin{array}{c}2.48 \pm 0.3 \\
\text { range }(2 \rightarrow 3)\end{array}$ & $\begin{array}{c}20(24) \\
10(1)\end{array}$ & $\begin{array}{c}13.8 \pm 1.5 \\
\text { range }(8-16)\end{array}$ & $\begin{array}{c}37.07 \pm 10 \\
\text { range }(30 \rightarrow 120)\end{array}$ \\
\hline$P$ value & & & & 0.6 & 0.08 & 0.1 & 0.6 \\
\hline
\end{tabular}

Table (8): Shows the number of stents used per infarct related artery

\begin{tabular}{|c|c|c|c|}
\hline No of stents & Group I & Group II & P value \\
\hline 1 & 22 & 25 & $>0.05$ \\
\hline 2 & 6 & 4 & $>0.05$ \\
\hline$\geq 3$ & 2 & 1 & $>0.05$ \\
\hline Total & 41 & 34 & $>0.05$ \\
\hline
\end{tabular}

Table (9): Shows the size, length, inflation pressure and duration of stent deployment in both groups.

\begin{tabular}{|l|c|c|c|c|}
\hline \multicolumn{1}{|c|}{ Groups } & Size $(\mathrm{mm})$ & Length $(\mathrm{mm})$ & Pressure (bar) & Duration $(\mathrm{sec})$ \\
\hline \multirow{2}{*}{ Group I } & $\begin{array}{c}3.2 \pm 0.4 \\
\text { range }(2.5-4)\end{array}$ & $\begin{array}{c}18.46 \pm 6.8 \\
\text { range }(12-33)\end{array}$ & $\begin{array}{c}14.4 \pm 1.9 \\
\text { range }(10-18)\end{array}$ & $\begin{array}{c}3.5 \pm 7.7 \\
\text { range }(30-60)\end{array}$ \\
\hline \multirow{2}{*}{ Group II } & $\begin{array}{c}2.9 \pm 0.2 \\
\text { range }(5-3.5)\end{array}$ & $\begin{array}{c}20 \pm 6.6 \\
\text { range }(12-38)\end{array}$ & $\begin{array}{c}14 \pm 1.6 \\
\text { range }(12-18\end{array}$ & $\begin{array}{c}34 \pm 8.5 \\
\text { range }(30-62)\end{array}$ \\
\hline P value & 0.4 & 0.15 & 0.4 & 0.6 \\
\hline
\end{tabular}




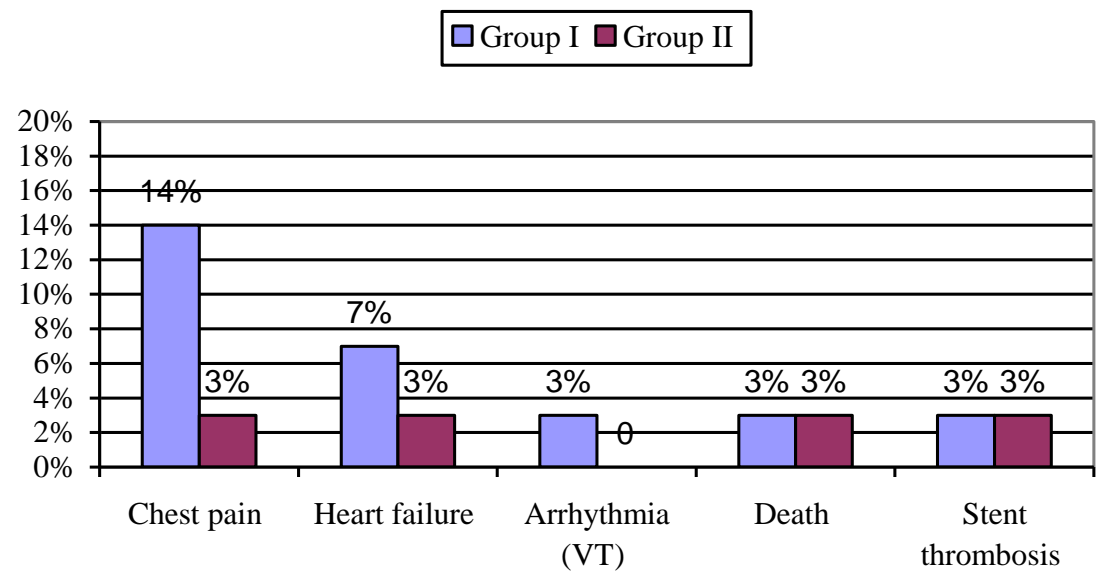

(P-value <0.05) (Only in chest pain recurrence).

Figure (3):Shows the in hospital course of patients in both groups.

Table (10): Shows the late follow up of patients in both groups (up to 6 months)

\begin{tabular}{|l|c|c|c|}
\hline & Group I & Group II & P value \\
\hline Recatheterization & $1(3 \%)$ (at 6 mo) & $1(3 \%)$ (at 1mo) & $>0.05$ \\
\hline CABG & $1(3 \%)$ at 3 mo) & - & $>0.05$ \\
\hline ICD implantation & $1(3 \%)$ (at 1 mo) & - & $>0.05$ \\
\hline
\end{tabular}

\section{Discussion}

Acute myocardial infarction is a potentially life threatening cardiovascular disorder. Revascularization is considered successful if done in the proper time namely in the first golden hour.

The patency rate of rt-PA at $90 \mathrm{~min}$ post MI onset is around $75 \%$ while the patency rate of primary PCI exceeds $90 \%$. The presenting study is comparing combining IV TNKase (metalyse) followed by primary PCI versus primary PCI alone in acute STEMI aiming at gaining the benefit of early revascularization by thrombolytic agent and high patency rate by the PCI to save as much as possible of the jeopardized myocardium

This approach although theoretically is sound yet many limitations of its use are present such as (1) it is restricted to the IV bolus of TNKase as it can be given outside hospital or in the Prehospital time window, (2) it increases the cost of the therapy for acute MI, (3) the risk of bleeding is more likely especially in the elderly and when glycoprotein IIb/IIIa inhibitors are concom- itantly used, (4) The decision to give the thrombolytic agent is left for the ER doctor who must be a cardiologist with adequate experience and complete awareness with other causes of acute chest pain with ST segment elevation.

In an attempt to limit bleeding complication in this study, the following precautions were undertaken: The very old patients were excluded ( $\geq 90$ years), careful right femoral artery puncture without transfixing the artery was carried out, the $5 \mathrm{~F}$ sheath was tried initially to avoid big hole in the wall of the artery $(60 \%$ of patients in group I versus $70 \%$ of patients in group II), before sheath removal PTT and clotting time were done and usually it is removed within 8 hours post PCI unless glycoprotein IIb/IIIa inhibitor was given, (in such case the sheath was left for 24 hours) and keeping in mind that all patients did not give past history of major bleeding.

TNKase facilitated revascularization of the infarct related artery in $80 \%$ of the patients which is close to the standard 


\section{Hussein Shaalan MD}

percentage $(75 \%)$. However, TIMI III flow was achieved only in $40 \%$ of patients which is less than the standard percentage $(63 \%)$ but similar to the results of ASSENT-4 study (43.5\%). This can be explained by the small number of the study population.

Clinically, chest pain disappeared after IV TNKase in only $33 \%$ of patients in group I. TIMI III flow was $0 \%$ in group II before PCI. (15\% in ASSENT-4) which enforces the importance of PCI post thrombolytic therapy.

The call to balloon time interval was longer in group I compared with group II (8.1 \pm 6.51 hours versus $4.57 \pm 3.3$ hours) (P $<0.05)$ which is explained by the farther distance of patients included in group I which highlights the importance of IV TNKase in those patients living away from the hospital.

Pathological Q waves were persistent in $50 \%$ of patients in group I versus $100 \%$ of patients in group II which probably reflect less myocardial damage in group I. However, the mean EF before and after PCI; and the peak enzyme level did not show statistical differences; this can be explained by more prevalence of inferior MI.

Tenecteplase did improved the heavy thrombus burden in group I (7\%) versus $(23 \%)$ in group II $(\mathrm{P}<0.001)$ taking into consideration that tirofiban was given in equal number of patients (43\%) in both groups.

These findings which facilitate revascularization enforce the added benefit of IV tenecteplase in acute STEMI before PCI.

Similarly, the mean procedural time of group I patients was $30.6 \pm 20 \mathrm{~min}$ versus $40 \pm 21$ min in group II (P value $<0.05$ ).

Although the angiographic success of PCI in group I was higher (97\%) compared with group II (93\%), p >0.05, the clinical success was lower $(67 \%)$ versus $(90 \%), p$ $<0.001$, which is an important limitation of the use of TNKase prior to PCI. Chest pain, heart failure and arrhythmias were more common among group I patients compared with group II. The reason for this paradox is not completely clear but might be related to more distal embolization of micro clots in the group receiving TNKase despite that significant number of patients underwent direct stenting, ( $\mathrm{P}<0.05)$, (Sabatier et al., 2002). However, the late follow up was not statistically different in both groups. Large volume trials are ongoing to address this issue.

These findings were concordant with ASSENT-4 study which was designed to determine whether immediate fibrinolysis prior to delayed PCI would eliminate the negative impact of that delay and improve clinical outcome. The study was prematurely stopped by the data and safety monitoring board (DSMB) due to a significant disadvantage in patients randomized to the facilitated PCI arm.

They concluded that immediate PCI following full-dose thrombolytic agent, as used in ASSENT-4 study cannot be recommended as a routine strategy.

Mortality rate in ASSENT-4 trial was higher in patients randomized at a hospital with a PCI facility and treated with thrombolysis as done in the presenting study. The call to balloon time was higher in group I compared with group II; (P <0.05), however the hard end points were not different which means that the delay in PCI was possibly overcomed by giving the thrombolytic agent in the first 2 hours post MI

The subgroup analysis in ASSENT-4 trial showed that patients with anterior wall infraction (the high risk group) and treated with TNKase plus PCI had higher mortality risk than those treated with PCI alone.

Similarly, the abciximab before direct angioplasty and stenting in myocardial infraction regarding acute and long-term follow-up (ADMIRAL trial) showed that patients pretreated with abciximab in the ambulance actually derived the most benefit whereas patients treated on-site did not.

The investigator of the GRACIA 2 trial concluded that catheterization plus adequate revascularization within 12 hours after the administration of facilitated fibrinolytic TNKase seems to be as safe and as effective as primary PCI.

These results suggest that both strategies are similarly effective in restoring myocardial perfusion, left ventricular function and both are associated with a 
beneficial clinical outcome.

Similarly, in the ADVANCE MI trial the study was prematurely terminated addressing the value of facilitated angioplasty after combination therapy or Eptifibatide monotherapy in acute myocardial infraction.

In the ADVANCE MI trial, all doses were reduced by approximately $25 \%$ in patients aged $\geq 75$ years. Combination therapy included aspirin, clopidogrel and either unfractionated heparin or LMWH enoxaparin.

The incidence of the combined primary end point of death or severe congestive heart failure was $11 \%$ in patients treated with eptifibatide plus tenecteplase and $1 \%$ in those treated with eptifibatide alone $(\mathrm{P}=0.02)$.

Bleeding complications were significantly higher in the group receiving eptifibatide plus tenecteplase than in the eptifibatide-only group.

Enrolment was stopped prematurely at 148 patients (aim was 5640 patients)

Previous studies had found that PCI following thrombolytic treatment was not beneficial and potentially harmful, but more recent experience using coronary stents and platelet glycoprotein IIb/IIIa inhibitors have found that PCI can be performed safely and effectively after pharmacologic reperfusion treatment. (Grines et al., 2003)

The investigators in the SPEED trial found that PCI following thrombolytic treatment or half dose thrombolytic treatment combined with platelet glycoprotein IIb/IIIa inhibitors was associated with superior outcomes compared to pharmacologic reperfusion treatment alone. (Herrmann et al., 2000)

The PAMI investigators and the Moses Cone Hospital Registry documented that patients undergoing primary PCI for AMI who arrive at the catheterization laboratory with an open versus a closed infract artery have higher procedural success rates, smaller infarct size, better recovery of left ventricular function, and lower early and late mortality. (Stone et al., and Brodie et al., 2000)

The major objective of facilitated PCI is to reduce the time delay inherent with mechanical reperfusion, but time to reperfusion appears to be less important with primary PCI than with thrombolytic therapy. (Brodie et al., 1998)

Also, combination pharmacologic treatment using half dose thrombolytics and platelet glycoproptein IIb/IIIa inhibitors has an increased bleeding risk and the same finite risk of intracranial hemorrhage as standard thrombolytic treatment as documented in the GUSTO V trial. (The GUSTO V investigators 2001)

In CADILLAC, ADMIRAL trials, when abcixiamab was given before PCI in acute MI, this was associated with fewer ischemic events and lower 30 day MACE.

The primary objective of reperfusion treatment for AMI has been the achievement and maintenance of brisk coronary flow (TIMI III flow); mechanical reperfusion combined with adjunctive treatment with stents and platelet glycoproptein IIb/IIIa inhibitors has achieved this objective with TIMI III flow rates of $>90$ $95 \%$, re infarction rates of $<1$, and late infarct artery re occlusion rates of $<5 \%$.

However, despite achieving very high rates of coronary reperfusion, recent data using sensitive markers of myocardial reperfusion, such as contrast echocardiography, myocardial blush assessment with angiography and electrocardiography ST segment resolution, indicate that $50 \%$ of patients with TIMI III flow fail to achieve optimal myocardial reperfusion, and these patients have increased complications and worse outcomes (Van'd Hof et al., 1997).

The reasons for that are likely to include distal embolization of platelet micro thrombi and micro circulatory dysfunction.

The presented study is similar to the CAPTIM trial (comparison of angioplasty and Prehospital thrombolysis in acute myocardial infarction) in which $70 \%$ of patients treated by thrombolysis underwent PCI up to day 30 , and $33 \%$ had urgent PCI (26\% labeled rescue PCI because of persistent ischemia). Therefore, CATIM really compares primary PCI to a strategy of Prehospital thrombolysis transfer to an interventional center, and subsequent PCI. This strategy is therefore very different from thrombolysis per se and probably 


\section{Hussein Shaalan MD}

explains why the results achieved by Prehospital thrombolysis in CATIM are very different from studies reported in the meta-analysis of Prehospital versus hospital thrombolysis.

In this trial, STEMI patients randomized within 2 hours after symptom onset to Prehospital thrombolytic therapy had a strong trend toward lower mortality and had a markedly lower rate of cardiogenic shock than patients treated with primary PCI. This difference was not apparent among patients randomized later.

This suggests that time since onset of symptoms should be considered when one selects reperfusion therapy. Prehospital thrombolysis with transfer to an interventional facility and, if needed, PCI is a valid treatment option and may even be preferable to primary PCI for patients treated early after symptoms onset. (American Heart Association, 2003).

The superiority of primary PCI over fibrinolysis appears not always to be present in a small but important subgroup of patients, those who can receive treatment in the first 2 hours after symptoms onset. (As shown in CATIM trial).

Because aging thrombi become more resistant to lyses, the efficacy of fibrinolytic therapy in establishing reperfusion and salvaging ischemic myocardium falls off with time from symptom onset, whereas the efficacy of PCI in achieving complete reperfusion and salvaging ischemic myocardial is far less time-dependent (Schoming et al., 2003 and Brodie et al., 1998).

\section{Conclusion}

The ideal reperfusion strategy could deliver rapid, complete, and sustained reperfusion with normalization of micro vascular flow.

Combining pharmacologic and catheterbased reperfusion strategies may accomplish these goals.

Early studies failed to demonstrate a benefit of routinely performing angioplasty early after thrombolysis (Michels et al., 1995)

However, these trials were carried out before the use of stents, glycoprotein IIb/IIIa inhibitors and fibrin specific thrombolytic agents (de Lemos 2001)

The results of FINESSE are still ongoing which address the issue of facilitated intervention with enhanced reperfusion speed to stop event.

To date facilitated angioplasty with thrombolytics with or without glycoprotein $\mathrm{IIb} / \mathrm{III}$ a was not demonstrated benefit in clinical trials and in the presenting study (no difference in the hard end point). Results of FINESSE and CARESS may show benefit.

The current ACC/AHA guidelines do not support the practice of routine PCI following the administration of thrombolytics with successful reperfusion. In developing countries such strategy has great limitations because of the significant burden on the national income.

\section{References}

1. Assessment of the Safety and Efficacy of a New Thrombolytic Regimen (ASSENT)-3 Investigators (2001): Efficacy and safety of tenecteplase in combination with enoxaparin, abciximab, or unfractionated heparin: The ASSENT-3 randomized trial in acute myocardial infarction. Lancet., 358:605,

2. Brodie BR and Stuckey TD (2002) : Mechanical reperfusion therapy for acute myocardial infarction: Stent PAMI, ADMIRAL, and CADILAC AND BEYOND. Heart., 87: 191 - 192.

3. Brodie BR, Stuckey TD, Hansen C, et al., (1998) : Importance of time to reperfusion for 30-day and late survival and recovery of left ventricular function after primary angioplasty for acute myocardial infarction. J. Am Coll Cardiol., 32: 1312- 19.

4. Brodie BR, Stuckey TD, Wall TC, et al. (1998) : Importance of time to reperfusion for 30- day late survival and recovery of left ventricular function after primary angioplasty for acute myocardial infarction. J. Am. Coll Cardiol., 32: 1312- 1319.

5. De luca G, Suryapranaa H, Zijlstra F, et al (2003) : Symptom-onset-to-balloon time and mortality in patients with acute myocardial infarction treated by primary angioplasty. J Am Coll Cardiol., 42:991,.

6. Dubois CL, Belmans A, Granger CB et al. (2003): Outcome of urgent and 
elective Percutaneous coronary interventions after pharmacologic reperfusion with tenectep-lase combined with unfractionated heparin, enoxaparin, or abciximab. J Am Coll Cardiol, 42:1178,

7. Ellis SG, Armstrong $\mathbf{P}$, Betriu A, Brodie B, Herrmann H, Montalescot G, Neumann FJ, Smith JJ, Topol E (2004: Facilitated Percutaneous coronary intervention versus primary Percutaneous coronary intervention: design and rationale of the facilitated intervention with enhanced reperfusion speed to stop events (FINESSE) trail. Am Heart J. Apr., 147 (4):E16.

8. Ellis SG, Da Silva ER, Spaulding CM, et al. (2000) : Review of immediate angioplasty after fibrinolytic therapy for acute myocardial infarction: Insights from the RESCUE I, RESCUE II and other contemporary clinical experiences. Am Heart J., 139: 1046,

9. Grines C, Serruys P, O'Neil WW (2003) : Fibrinolytic therapy: is it a treatment of the past? Circulation, 107: 2538- 2543.

10. Grupo de Análisis de la Cardiopatia Isquémica Agude (GRACIA). (2002) : Randomized trial comparing stenting within 24 hours of thrombolysis versus ischemia-guided approach to thrombolysed acute myocardial infraction with ST elevation. F. Fernandez- Avilés, the Annual Meeting of the European Society of Cardiology, August 31,

11. Hermann HC, Moliterno DJ, Ohman EM., et al. (2000) : Facilitation of early Percutaneous coronary intervention after releplase with or without abciximab in acute myocardial infarction results from the SPEED (GUSTO-4 pilot) trial J Am Coll Cardiol ., 36: 1989-1996.

12. Michels KB, Yusuf S (1995) : Dose PTCA in acute myocardial infarction affect mortality and re infarction rates? A quantitative overview (meta-analysis) of the randomized clinical trails. Circulation 91: 476-485.

13. Montalescot G, Barragan B Wittenberg O, et al. (2001): Platelet glycoprotein
IIb/IIIa inhibition with coronary stenting for acute myocardial infarction. N Engl J Med 344:1895.

14. Montalescot G, Barragan T, Wittenberg O. et al. (1903) : for the ADMIRAL Investigators. Platelet glycoprotein IIb/IIIa inhibition with coronary stenting for acute myocardial infection. N Eng1 J Med. 344: 1895-.

15. Morrison Lj, Verbeek PR, McDonald AC, et al (2000) : Mortality and Prehospital thrombolysis for acute myocardial infarction: A meta-analysis. JAMA., 283:2686.

16. Nallamothu BK, Bates ER: (2003): coronary intervention versus fibrinolytic therapy in acute myocardial infarction: Is timing (almost) everything? Am J Cardiol 92:824, 2003.

17. Philippe Abriel Steg, Eric Bonnefoy, Sylvie Chaayd frédéric Lapostolle, Pierre-Yves Dubien, Pascal Crisofini, Alain Leizorovicz, Paul Tobol : (2003) . The Comparison of Angioplasty and Prehospital Thrombolysis In acute Myocardial infarction (CAPTIM) Investigators. Impact of time to treatment on mortality after Prehospital fibrinolysis or primary angioplasty data from the CAPTIM randomized clinical trial. Circulation ., 108; 2851-2856.

18. Sabatier R, Hamon M, Zhao QM, et al (2002): Could direct stenting reduce noreflow in acute coronary syndromes? A randomized pilot study. Am. Heart. J., 143:1027,

19. Stone GW, Cox D, Garcia E et al. (2000): Normal flow (TIMI-3) prior to mechanical reperfusion therapy is an independent determinant of survival in acute myocardial infraction - analysis from the PAMI trial. Circulation (in press).

20. Van't Hof AWJ, Liem A, de Boer MJ, et al. (1997) : Clinical value of 12 lead electrocardiogram after successful reperfusion therapy for acute myocardial infarction. Lancet., 350: 615-19. 


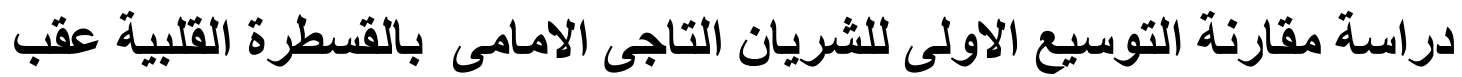

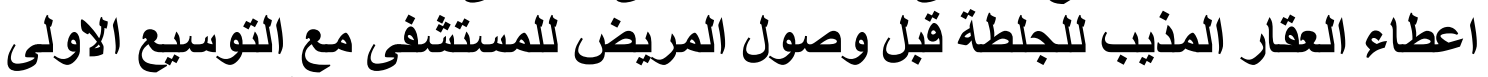

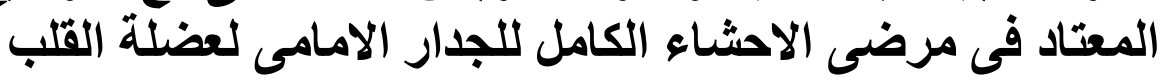

\author{
حسين شعلان \\ قسم القلب - جامعة عين شمس شئ
}

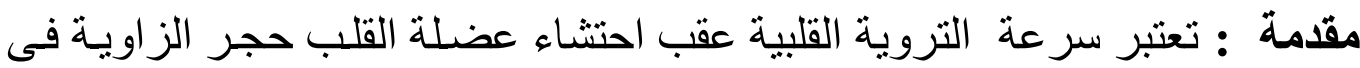

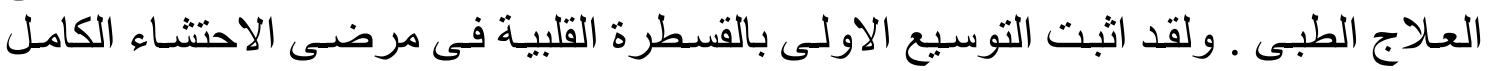

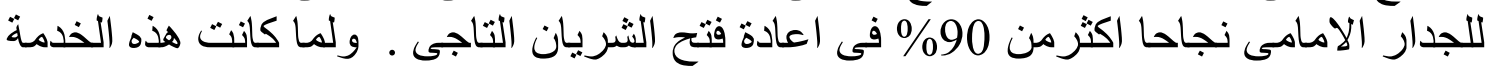

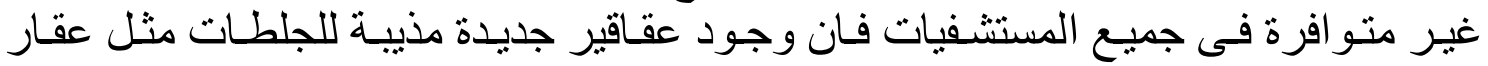

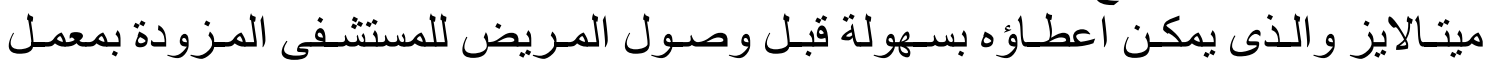

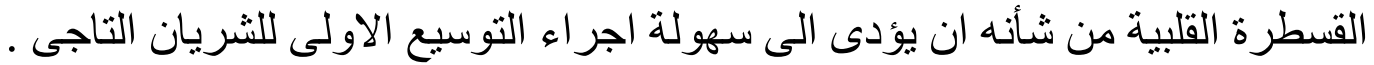

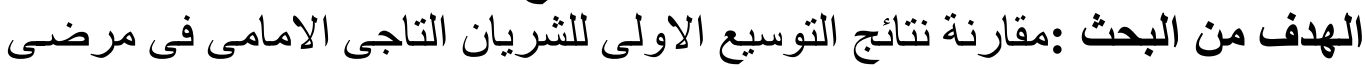

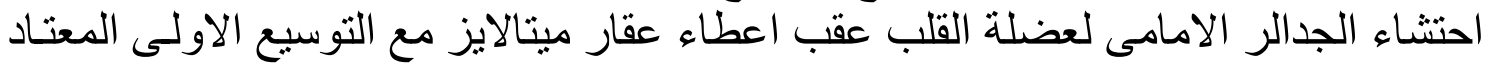

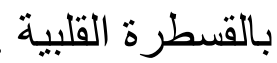

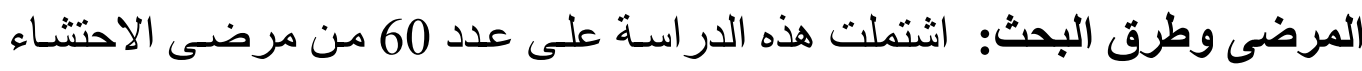

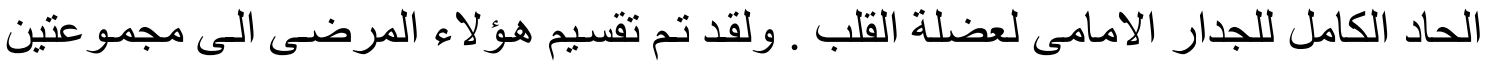

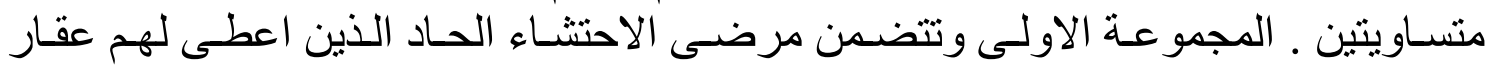

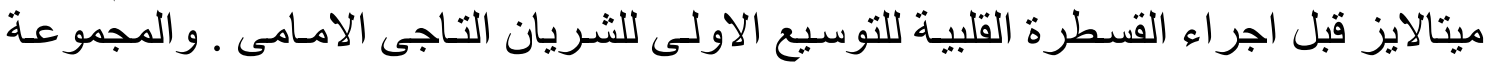

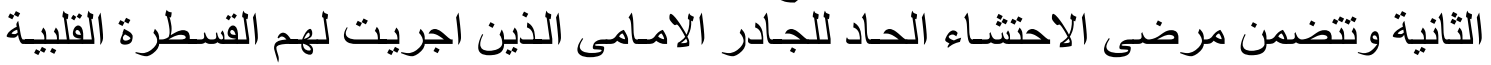

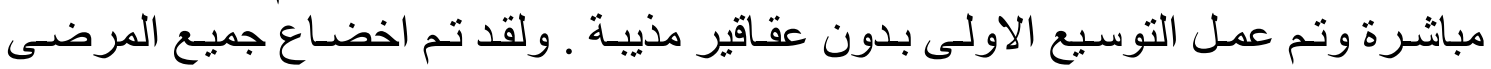

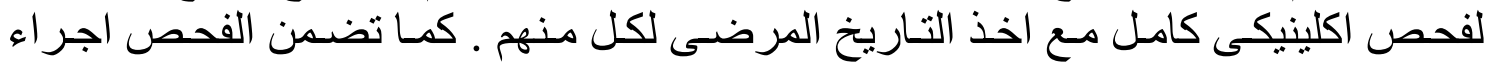

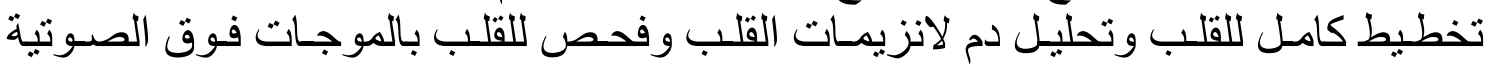

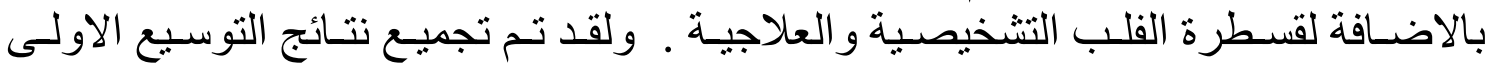

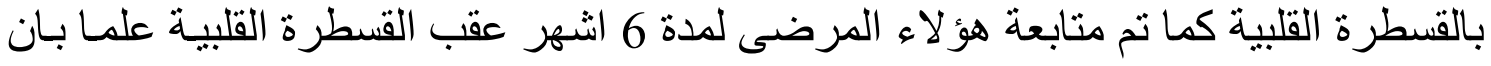

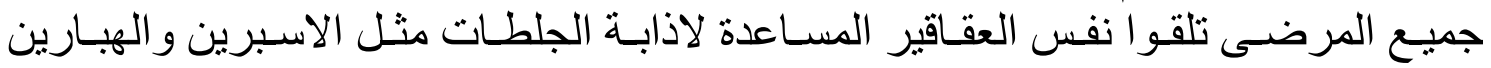

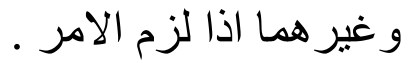

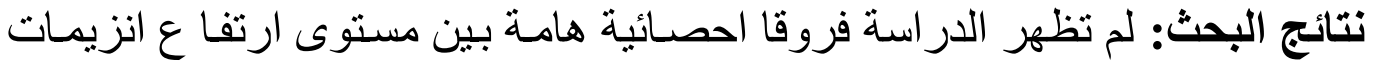

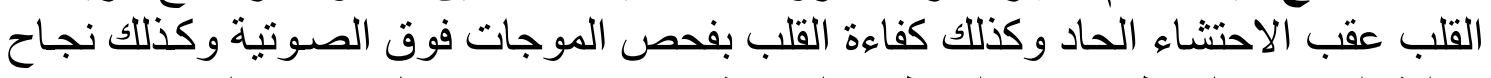

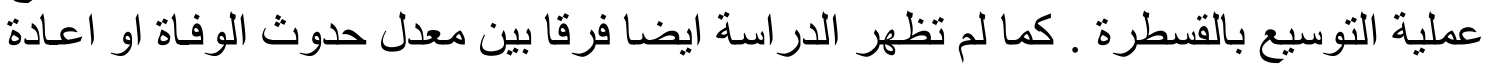

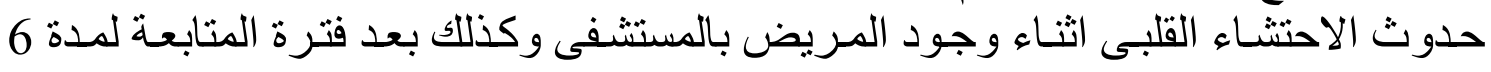

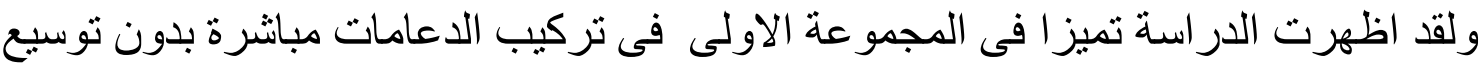

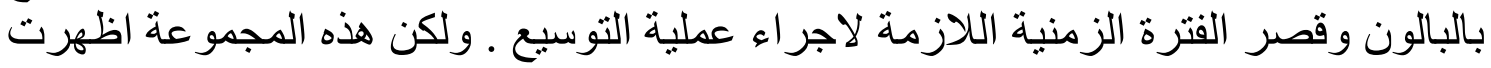

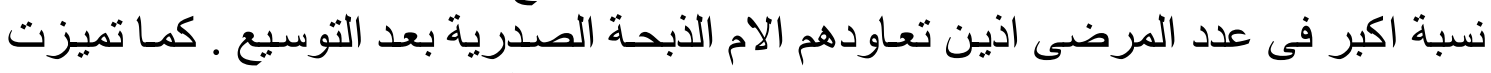

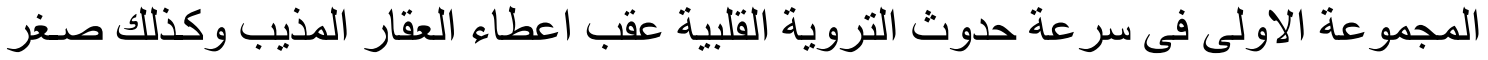

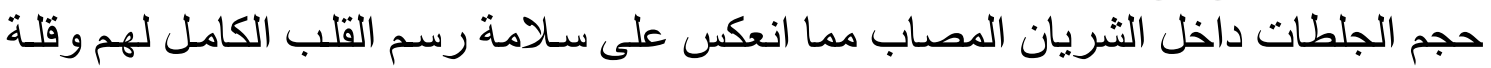


وجود التغير ات المرضية به والذى لم يختلف كثثر ا عن تخطيط القلب قبل حدوث الاحتشـاء الحاد.

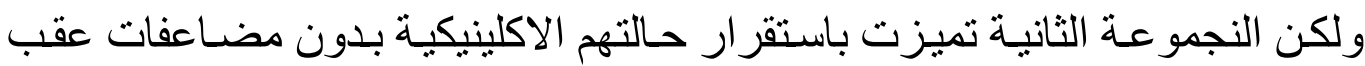

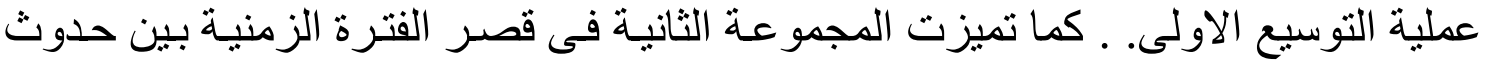

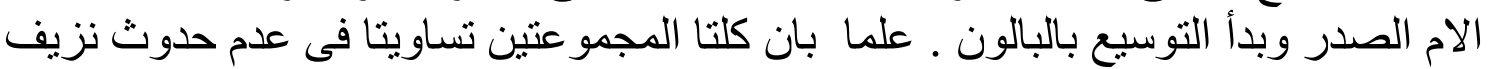

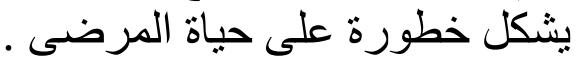

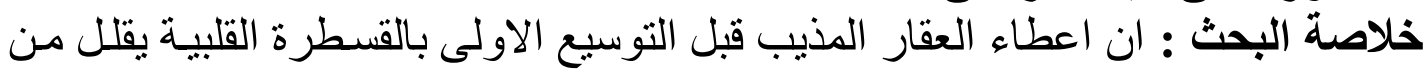

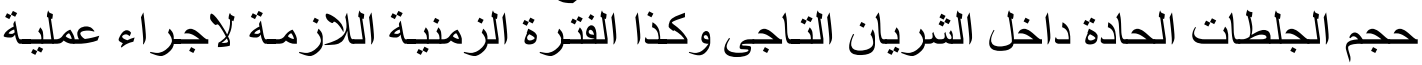

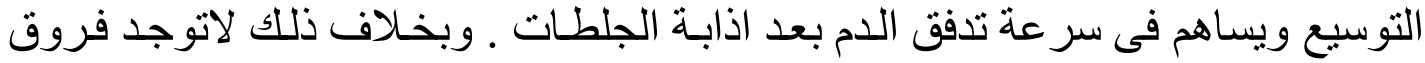

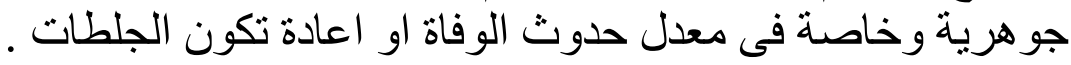

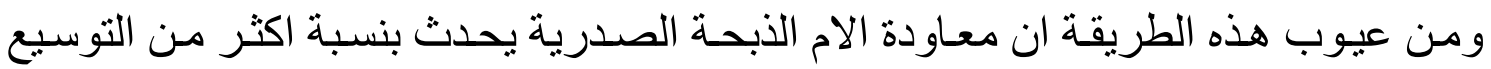

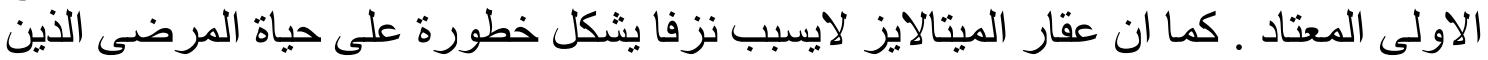

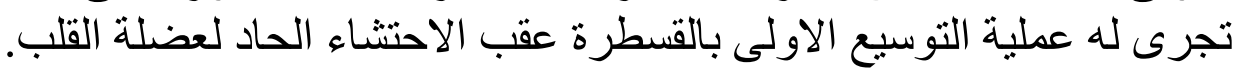

